\title{
Briefe an die Redaktion
}

Zum Artikel „Reisen mit Rollstuhl“ (ergopraxis 7-8/11)

\section{Auf den Punkt gebracht}

Sehr geehrte Frau Gritsch,

da ich zwar zum Kreise der medizinisch-technischen Dienste gehöre, aber nicht Ergotherapeutin bin, bin ich erst heute - und das auch nur durch Zufall - auf Ihren Artikel gestoßen. Da ich selbst seit 22 Jahren auf den Rollstuhl angewiesen bin und leidenschaftlich gerne reise, war es für mich schon fast ein Muss, Ihren Bericht zu lesen.

Kurz und prägnant gesagt: Ich war begeistert! Endlich jemand, der unsere Problematik tatsächlich zu verstehen scheint! Ihr Bericht trifft RollifahrerInnen mitten ins Herz, denn wie oft versucht jeder von uns, seinen Mitmenschen irgendwie klarzumachen, dass die meisten von uns nicht bösartig sind, sondern wir einfach nur nicht behindert werden wollen. Nicht nur, dass Sie verständnisvoll und einfühlsam die Probleme von RollstuhlfahrerInnen auf Reisen auf den Punkt bringen, Sie

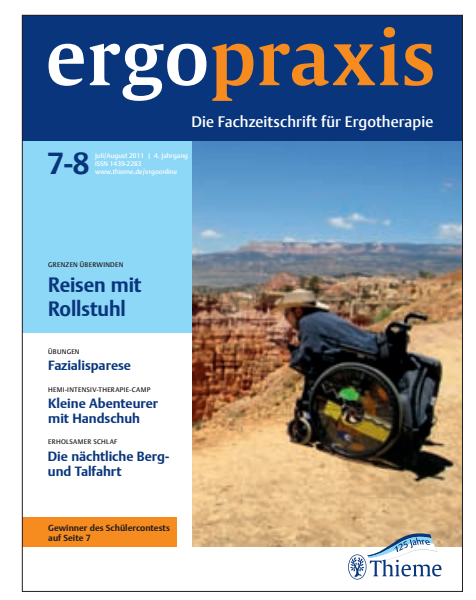

nennen auch Beispiele, wie der Artikel 3 des Grundgesetzes ,Jeder hat das Recht auf Teilhabe" mit ein bisschen gutem Willen von Seiten der Verantwortlichen ganz leicht verwirklicht werden könnte. Vielleicht verstehen Sie nun meine Begeisterung über Ihren Artikel.

Einen kleinen Kritikpunkt möchte ich dennoch anbringen, der Ihren letzten Satz betrifft:
„All diese Dinge bedeuten viel für einen Rollstuhlfahrer und schaden keinem Fußgänger. Im Gegenteil. Fußgänger gehen in Zügen gern auf die behindertengerechte Toilette. Kein Wunder - die ist so schön groß.“ Diese Passage macht leider den positiven Eindruck, den ich beim Lesen hatte, beinahe zunichte. Realität ist, dass RollstuhlfahrerInnen fast immer damit zu kämpfen haben, dass Behinderten-WCs entweder kaputt, total verschmutzt oder gerade von einer Schlange Menschen belagert sind, die alle auf das „ach so schöne große WC“ wollen. Jeder will heute offensichtlich ein kleines bisschen behindert sein, um damit das Recht auf die Benutzung von Rollstuhlparkplätzen und Behinderten-WCs einfordern zu können.

Es gibt sicher Menschen, die den letzten Teil Ihres Berichtes als Freibrief sehen, um so weitermachen zu können wie bisher, denn wenn es sogar in einer Zeitschrift so quasi schwarz auf weiß steht, dass Rolli-WCs eine super Sache für Geherlnnen sind, ja dann machen sie ja alles richtig, wenn sie „unsere“

\section{GEWINNER AUS ERGOPRAXIS 11-12/11}

Dank Sponsoren gibt es in jeder Ausgabe von ergopraxis etwas zu gewinnen. Kästen innerhalb der Artikel weisen auf die Gewinnspiele hin. Einfach bis zum Ablauf der Frist das gewünschte Stichwort bei www.thieme.de/ergoonline > „ergopraxis“ > „Gewinnspiel“ anklicken. Viel Glück!

Kursplatz

„Grundkurs „Warnke-Verfahren“““: Brigitte Heinlein aus Nürnberg

Bücher

„Frühphase Schlaganfall“: Insa Kruse aus Tarmstedt, Stefanie Straßner aus Sankt Martin, Sonja Kläner aus Neerstedt

„Ergotherapie bei Kindern mit ADHS“: Leticia P. Medici aus Stuttgart, Lisa Bücker aus Oelde

Notizbuch „Steine“: Theresa Pulsfort aus Marktredwitz, Cornelia Putnaerglis aus Bremen, Regina Gaida aus Wien (Österreich), Michaela Zakowski aus Grenzach-Wyhlen „Das neue große Buch der Trennkost“: Doreen Herbarth aus Rohr, Heidrun Schröder aus Wedel, Nina Unger aus Heidelberg

und außerdem

Set Hot Chocolate: Linda Kohlmann aus Berlin

Tanzsäcke: Brigitta Schmeißer aus Berlin, Cassandra Römer aus Molsdorf

Business Timer: Rita Pieperhoff aus Dortmund, V. Kröhle aus Braunschweig, Mona Gallo aus Nürnberg

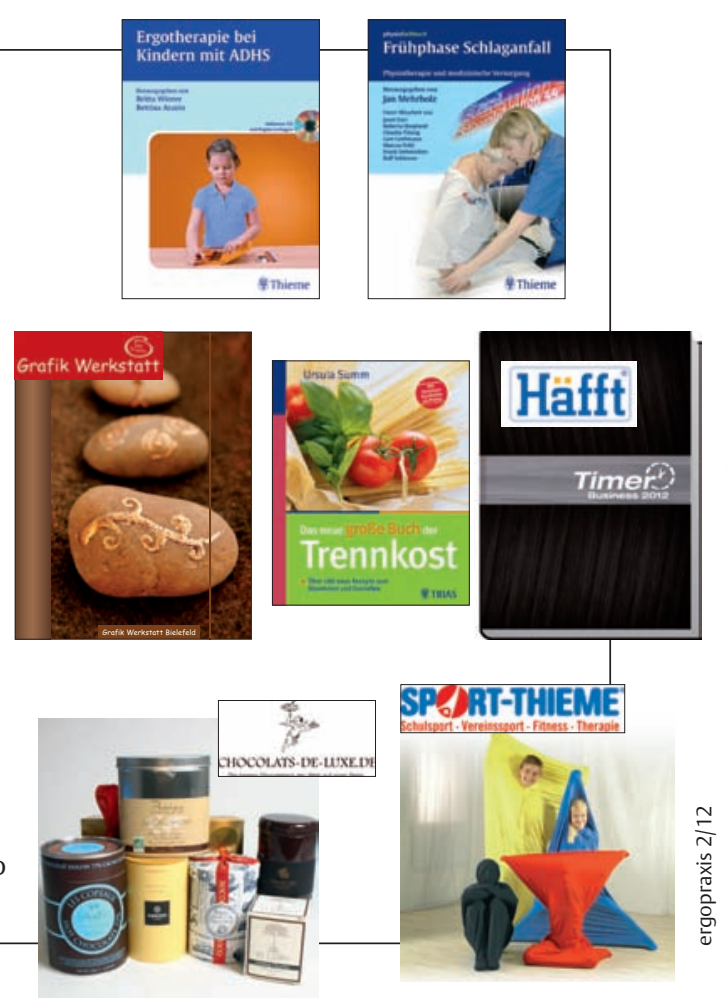




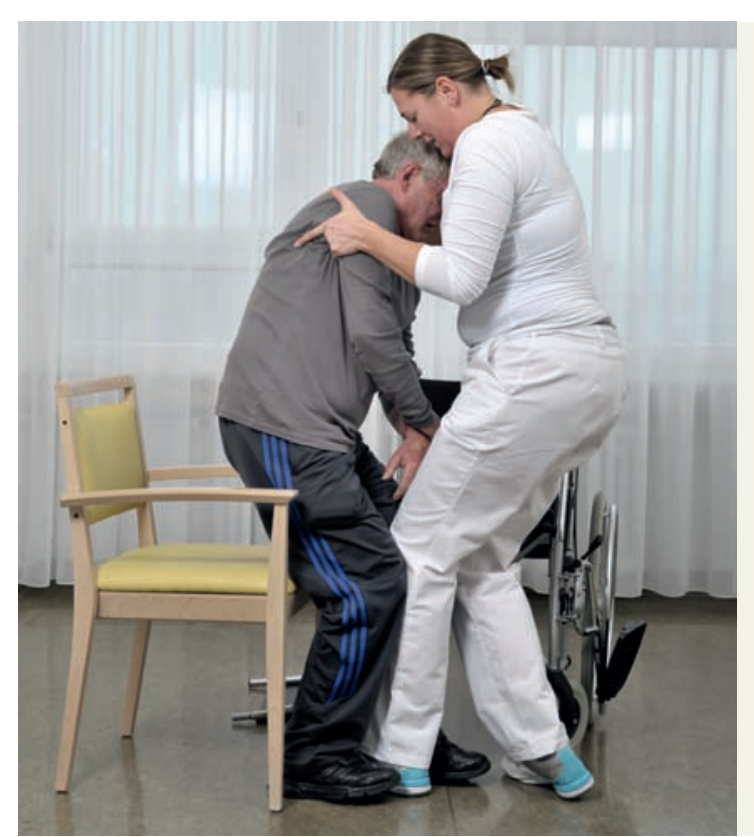

Unterstützen Sie ihn weiter an den Schultern beim Aufstehen und sichern Sie weiterhin seine Knie.
Toiletten benützen. Als Betroffene, aber auch als Kollegin, ist es mir ein großes Anliegen, Sie dafür zu sensibilisieren, dass Sie bei einem Ihrer nächsten, sicher genauso tollen und einfühlsamen Berichte deren Wert nicht durch einen einzigen unbedachten Satz schmälern.

\section{Herzliche Grüße}

Annemarie Grillenberger aus Wien (Österreich)

Zum Artikel „Transfers bei Patienten mit Hemiplegie“ (ergopraxis 1/12)

\section{Skapula schonen}

Liebes ergopraxis-Team,

da ich im Bereich Neurologie/Geriatrie tätig bin und auf Honorarbasis neurophysiologische Behandlungsverfahren unterrichte, habe ich mit Freude den Artikel sehr genau durchgelesen. Hier allerdings ist mir etwas Negatives aufgefallen: Als Therapeutin sollte ich vom Sitz in den Stand niemals unter den betroffenen Arm des Klienten greifen, da es dadurch zu negativen Auswirkungen an der Skapula und dem betroffenen Arm kommen kann. Der Klient benötigt eine deutliche Fazilitation am Gesäß, um leichter in den Stand zu kommen.

Beim Transfer Rollstuhl-Auto macht der Klient einen „schönen“ alltäglichen Fehler, mit dem wir oft zu kämpfen haben: Er fasst den Therapeuten um den Hals, was wiederum auf die Wirbelsäule des Therapeuten sehr negative
Folgen haben kann. Ich wünsche trotzdem weiterhin gutes Gelingen - und weiter so!

Herzliche Grüße

Anika Cordes aus Reeßum

\section{Mikrotraumen möglich}

Sehr geehrte Damen und Herren, auf Seite 30 wird ein „Stuhl-Rollstuhl-Transfer" über die betroffene Seite mit Fotos erklärt (๑ Abb.). Hier werden beide Hände unter den Schultern bzw. unter den Achseln positioniert, wobei es beim Transfer zwangsläufig zu einer Elevation der betroffenen Ska-

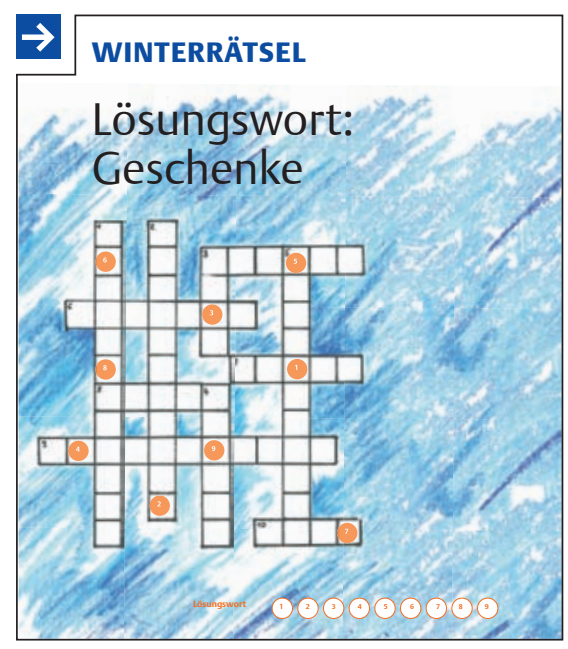

pula mit Beteiligung im GHG kommt. Bei einer Subluxation des Schultergelenks, das heißt bei etwa $80 \%$ aller hemiplegischen Patienten in der Frühphase, führt ein derartiges Handling zu Mikrotraumen und Einklemmungen von Weichteilen im Schulterbereich, wodurch Schmerzen, chronische Schmerzen und im schlimmsten Fall ein Schulter-Hand-FingerSyndrom entstehen können.

In Reha-Einrichtungen wird genau dieses Handling kritisiert, und Alternativen für Pflegepersonal und Angehörige werden erarbeitet. Ein derartiges Handling ist nicht "sicher“, sondern gefährdet den Reha-Verlauf des Patienten in Bezug auf die betroffene obere Extremität. Gerade Berufsanfänger stützen sich auf derartige Artikel und hinterfragen sie häufig nicht.

Mit freundlichem Gruß

Max Helber aus Graz (Österreich)

\section{Anmerkung der Redaktion}

Liebe Frau Cordes, lieber Herr Helber,

vielen Dank für Ihr Feedback! Im Falle der angesprochenen Bilderstrecke handelte es sich um einen fitten Patienten, der lediglich ein Initial an den Schultern in die richtige Richtung bekam. Wir stimmen Ihnen voll und ganz zu, dass an dieser Stelle Vorsicht geboten ist.

Beim unterstützten Transfer sollte die Therapeutin auf keinen Fall von vorne unter die Achseln des Patienten greifen und daran nach oben ziehen. „Hands on“ im Bereich von Schulter und Skapula unterstützt lediglich die Verlagerung des Patienten nach vorne. Ist mehr Unterstützung nötig, könnte die Therapeutin beispielsweise seitlich stehen und das Aufstehen zusätzlich am Gesäß fazilitieren.

!

\section{SCHREIBEN SIE UNS}

Das Leserforum ist Ihre Seite für fachlichen Austausch. Meinungen, Kommentare und Anregungen sind willkommen! Wir behalten uns vor, die Briefe zu kürzen. Die Texte spiegeln die Meinung des Verfassers wider und nicht die der Redaktion. Leserbriefe an:

Georg Thieme Verlag KG

Redaktion ergopraxis

Rüdigerstraße 14

70469 Stuttgart

Fax: 0711/8931-871

E-Mail: ergopraxis@thieme.de 